\title{
Inducible Clindamycin resistance among clinical isolates of Staphylococcus aureus at a Rural tertiary care teaching hospital of western Uttarpradesh
}

\section{Abhishek Mehta *, Vijay Prakash Singh.}

Department of M icrobiology, K.D. Medical College Hospital \& Research Center , M athura, Uttar Pradesh, India.

\section{ABSTRACT}

Background: Staphylococcus aureus a notorious pathogen is rapidly acquiring resistance against most of the major group of antibiotics including Clindamycin. Inducible Clindamycin resistance is difficult to detect and is often missed in routine antibiotic susceptibility testing. Prompt and timely detection of such resistance in the clinical isolates of S.aureus is imperative to formulate appropriate strategy for the effective treatment of such infections.

Aims \& Objectives: To determine the percentage of inducible Clindamycin resistance among clinical isolates of S.aureus in our geographical area using D-test and to distinguish different susceptibility patterns/resistance phenotypes in Erythromycin resistant clinical isolates of S.aureus.

Materials and Methods: A total of 244 consecutive non-duplicate isolates of S.aureus recovered from variousclinical specimens(Urine,pus, wound swab,blood,body fluids,aspirates etc.) were subjected to Antibiotic Susceptibility Testing by Standard disc diffusion method and M ethicillin resistance testing using Cefoxitin $\operatorname{disc}\left(30 \frac{1}{4 \mathrm{gm}}\right)$. Erythromycin resistant S.aureus isolates were subjected to D-test as per CLSI 2014 guidelines.

Result: Out of 244 clinical isolates of S.aureus, $49(20 \%)$ were found to be MRSA and $195(80 \%)$ M SSA strains.74(30.33\%) were Erythromycin resistant and were subjected to D-test.16 isolates(6.56\%) exhibited

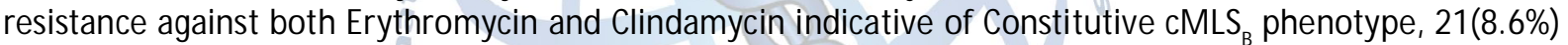
were found to be D-test positive for inducible resistance(iM $\mathrm{LS}_{\mathrm{B}}$ phenotype) while 39(15.98\%) were D-test negative and hence truly susceptible to Clindamycin(M S phenotype).

Conclusion: The observations of this study had clearly revealed that if D-test would've not been performed then a significant proportion of Erythromycin resistant isolates would have been reported wrongly as Clindamycin sensitive and this could have resulted in the treatment failure.So,D-test must be incorporated in routine Antibiotic susceptibility testing for S.aureus isolates so as to ensure the judicious and rational use of this valuable drug. KEY WORDS: D-Test, M ethicillin Resistant Staphylococcus aureus (M RSA), M ethicillin Sensitive Staphylococcus aureus (M SSA), Constitutive M acrolide Lincosamide Streptogramin B Phenotype (cM LS $S_{B}$ ), Inducible M acrolide Lincosamide Streptogramin B Phenotype $\left(\mathrm{iM} \mathrm{LS}_{\mathrm{B}}\right)$, M S phenotype.

Address for correspondence: Dr.Abhishek M ehta, Department of M icrobiology, KD M edical College, Hospital \& Research Center, 24 Km. milestone, NH\#2, AKBARPUR-281406, Distt.M athura (UP), India. Phone: +91-9897620394 E-M ail: abhishekmehta623@gmail.com

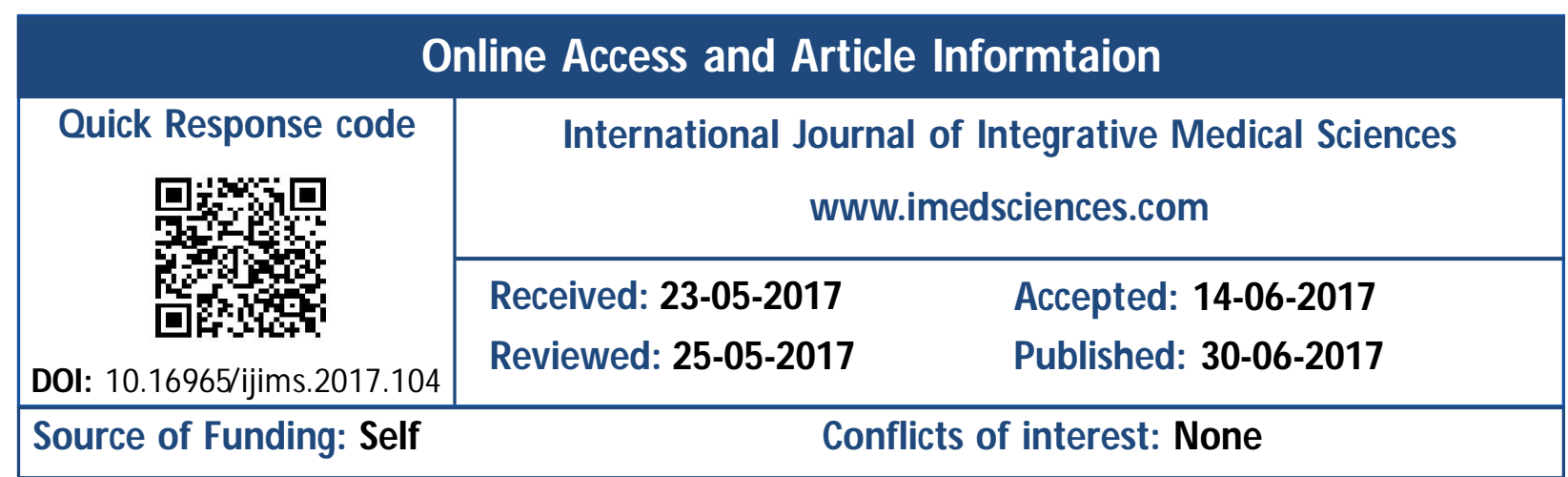

\section{BACKGROUND}

Staphylococcus aureus has been recognised throughout the world as one of the most common pathogen causing a wide range of nosocomial as well as community acquired infections [1]. But such infections are now rapidly emerging as a grave threat to the public health as the 
pathogen is rapidly acquiring resistance against most of the major group of antibiotics being rendered refractory to therapy leading to treatment failure. Clinicians are struggling hard to treat such infections with very few alternatives left in their arsenal.

One such alternative left which is quiet effective and preferred by clinicians in treating such infections is Macrolide-Lincosamide-Streptogramin (M LSB) group of antibiotics. In this group a lincosamide Clindamycin has emerged as the most popular and most frequently used option due to its excellent pharmacokinetic properties, $100 \%$ bioavailability on oral administration, good tissue penetration, tendency to accumulate in abscesses, tolerability and low cost [1-4].

However it has been observed that resistance towards Clindamycin can develop in S.aureus strains. This resistance is mainly attributed to the erm gene mediated target site modifications which can be either Constitutive or Inducible [36].

Inducible Clindamycin resistance in iM LSB phenotypes is not easy to detect and is often missed as the isolates appear Clindamycin sensitive but erythromycin resistant in vitro if their discs are not placed adjacent to each other in Standard disc diffusion test[1].

Treatment of such cases with Clindamycin leads to selection of Constitutive erm mutants (CM LSB phenotypes) further worsening the situation [16].

But there is another mechanism of resistance towards this group mediated via msrA genes through efflux of antibiotics which presents apparently a similar picture where isolates appear Clindamycin sensitive but erythromycin resistance not only in vitro but also in vivo(MS phenotypes) and respond well to Clindamycin therapy [1,2].

Prompt and timely detection of inducible Clindamycin resistance in the clinical isolates of S.aureus is becoming increasingly important to formulate appropriate strategy for the effective treatment of such infections.

In the light of above facts we had undertaken this study to determine the percentage of inducible Clindamycin resistance among clinical isolates of S.aureus in our geographical area using D-test.

\section{MATERIALS AND METHODS}

This cross-sectional study was conducted in the Dept. of M icrobiology of a rural tertiary care teaching hospital of western Uttarpradesh from Jan.2016 to Dec.2016 for a duration of one year. A total of 244 consecutive non-duplicate isolates of S.aureus recovered from various clinical specimens(Urine, pus,wound swab,blood,body fluids, aspirates etc.) sent to Bacteriology Lab. for routine culture sensitivity as a part of standard diagnostic protocol. All the samples were processed using Standard microbiological techniques [7].

Antibiotic Susceptibility Test [8]: In vitro antibiotic sensitivity test for all S.aureus isolates was conducted by Kirby Bauer Standard Disc diffusion method on MHA plates as per CLSI-2014 guidelines using following antibiotic discs:

1)Amoxycillin, 2)Erythromycin, 3)Clindamycin 4)Cefoxitin, 5)Doxycyclin, 6)Gentamycin, 7)Ciprofloxacin/Norfloxacin, 8)Cotrimoxazole

Methicillin Resistance Test: Test for mecA mediated Oxacillin resistance [8]: Those S.aureus isolates which exhibited resistance to Cefoxitin disc $(30 \mu \mathrm{g})$ with Zone of inhibition diameter $\leq 21 \mathrm{~mm}$ by Standard disc diffusion method as per CLSI guidelines were reported as MRSA strains.

\section{D-Zone Test for screening Inducible} Clindamycin resistance in S.aureus isolates[1,2,3,6,8,9,10]: All the clinical isolates of S.aureus which were found to be resistant to Erythromycin $(15 \mu \mathrm{g})$ by Standard disc diffusion method were further tested for inducible Clindamycin resistance by D-Zone test as per CLSI-2014 guidelines.

0.5 M cFarland standard suspension of test strains inoculated on prewarmed MHA plates. Erythromycin $(15 \mu \mathrm{g})$ and Clindamycin $(2 \mu \mathrm{g})$ discs were placed over the seeded plate spaced $24 \mathrm{~mm}$.apart edge to edge. After overnight incubation at $37^{\circ} \mathrm{C}$ for $18 \mathrm{hrs}$. 
Interpretation of D-Test was done as follows: 1) Inducible iM LS $S_{B}$ phenotype (D-test positive): Test strains of S.aureus exhibiting resistance against Erythromycin $(15 \mu \mathrm{g})$ with zone size $\leq 13 \mathrm{~mm}$. but being apparently sensitive to Clindamycin with zone size $\geq 21 \mathrm{~mm}$. and demonstrating $D$ shaped inhibition zone around Clindamycin disc with the flattening towards Erythromycin disc were considered to be D-test positive for inducible resistance( iM LS $_{B}$ phenotype).

2) MS phenotype(D-test Negative): Test strains of S.aureus showing resistance towards Erythromycin with zone size $\leq 13 \mathrm{~mm}$ but sensitive to Clindamycin with zone size $\geq 21 \mathrm{~mm}$, zones of inhibition being circular were considered to be D-test negative for inducible resistance(M S phenotype).

3) Constitutive $\mathbf{c M L S _ { B }}$ phenotype: Test strains of S.aureus exhibiting resistance towards both Erythromycin with zone size $\leq 13 \mathrm{~mm}$ and Clindamycin with zone size $\leq 14 \mathrm{~mm}$ with a circular zone of inhibition if any around Clindamycin.

Quality control of antibiotic discs was performed with S.aureus ATCC 25923 as per CLSI guidelines.

\section{RESULTS AND DISCUSSION}

Out of 244 clinical isolates of S.aureus, 49(20\%) were found to be M RSA and 195(80\%) were MSSA strains.

Out of all 244 isolates 74(30.33\%) were Erythromycin resistant and were subjected to Dtest.16 isolates(6.56\%) exhibited resistance against both Erythromycin and Clindamycin indicative of Constitutive $C M$ LS $_{B}$ phenotype.
Out of the 57 isolates which appeared to be sensitive to Clindamycin, 21(8.6\%) were found to be D-test positive for inducible resistance (iM LS B $_{\text {p }}$ henotype) while 39(15.98\%) were D-test negative and hence truly susceptible to Clindamycin(M Sphenotype).

Amongst M RSA strains Constitutive $\mathrm{CM} \mathrm{LS}_{B}$ phenotypes were $8.16 \%$ and Inducible $i M L_{B}$ phenotypes came out to be $12.2 \%$ while amongst MSSA strains CM LS $S_{B}$ phenotypes were $6.15 \%$ and iM LS $S_{B}$ phenotypes came out to be $7.69 \%$.

Erythromycin and Clindamycin Susceptibility patterns among S.aureus isolates and interpretation of D-test is depicted in Table-1 and Fig-1 respectively.

In this study we had found a significantly high percentage of Erythromycin resistant isolates(30.33\%) and amongst them $21(28.38 \%)$ were D-test positive for inducible Clindamycin resistance.

The observations of this study had clearly revealed that if $D$-test would've not been performed then a significant proportion of Erythromycin resistant isolates would have been reported wrongly as Clindamycin sensitive and this could have resulted in the treatment failure.

It has also been found in this study that the percentages of inducible and constitutive Clindamycin resistance were higher among M RSA strains( $12.2 \% \& 8.16 \%)$ as compared to MSSA strains $(7.69 \% \& 6.15 \%)$. This finding is in accordance with a number of other studies [1,4,12-14] (Yilmaz et al, Gadepalli et al, M ohammad Rahabar et al, Ajantha et al, Prabhu Ket al.).

Fig. 1: Interpretation of D-zone test for inducible Clindamycin resistance.

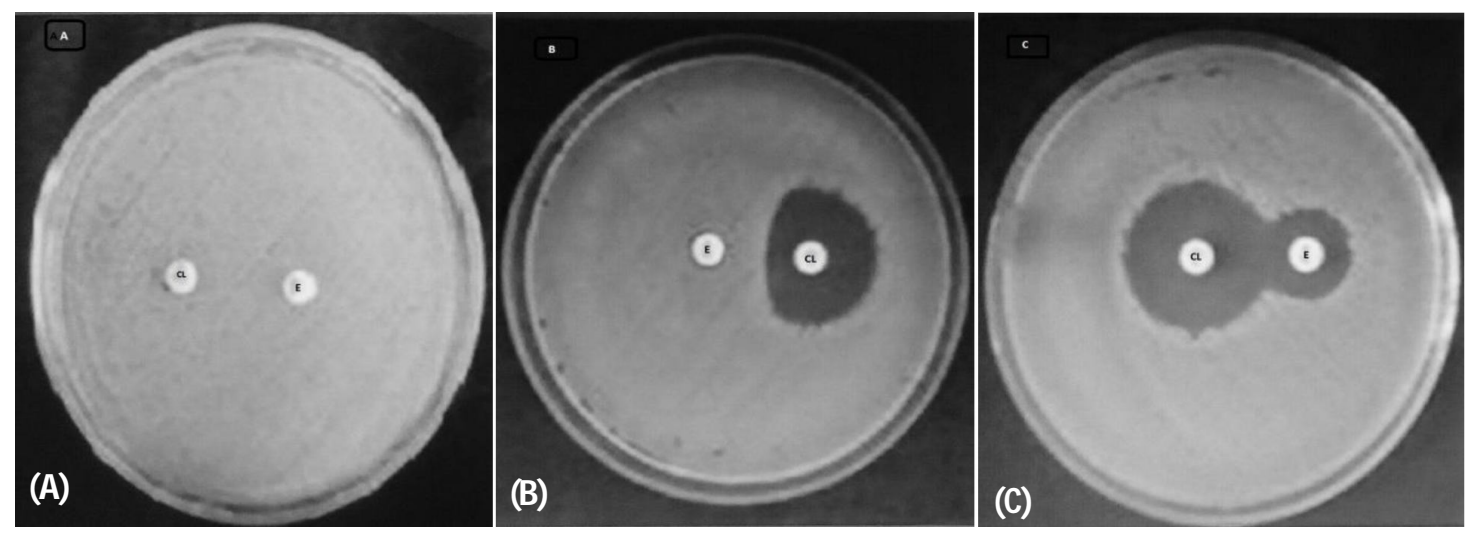

(A) Constitutive cM LSB phenotype, (B) Inducible iM LSB phenotype, (C) M S phenotype 
Table 1: Erythromycin and Clindamycin Susceptibility patterns among S.aureus isolates.

\begin{tabular}{|c|c|c|c|c|c|}
\hline \multirow{2}{*}{$\begin{array}{c}\text { Susceptibility } \\
\text { pattern }\end{array}$} & \multirow{2}{*}{ D-Test } & \multirow{2}{*}{ Phenotype } & \multicolumn{3}{|c|}{ No. of isolates (\%) } \\
\cline { 4 - 6 } & & & $\begin{array}{c}\text { MRSA } \\
(\mathrm{n}=49)\end{array}$ & $\begin{array}{c}\text { MSSA } \\
(\mathrm{n}=195)\end{array}$ & $\begin{array}{c}\text { Total } \\
(\mathrm{n}=244)\end{array}$ \\
\hline Ery-S Clind-S & & Susceptible & $32(65.3 \%)$ & $136(69.74 \%)$ & $168(68.85 \%)$ \\
\hline Ery-R Clind-R & Negative & $\mathrm{CMLS}$ & $4(8.16 \%)$ & $12(6.15 \%)$ & $16(6.56 \%)$ \\
\hline Ery-R Clind-S & Positive & $\mathrm{i}$ MLSB & $6(12.2 \%)$ & $15(7.69 \%)$ & $21(8.6 \%)$ \\
\hline Ery-R Clind-S & Negative & MS & $7(14.3 \%)$ & $32(16.41 \%)$ & $39(15.98 \%)$ \\
\hline
\end{tabular}

Ery-Erythromycin, Clind- Clindamycin, s- Susceptible, R-Resistant, D-Test: Disc Approximation Test, MRSA- M ethicillin Resistant Staphylococcus aureus, MSSA- Methicillin Sensitive Staphylococcus aureus,

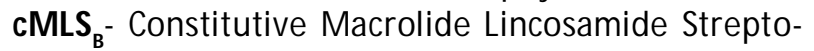

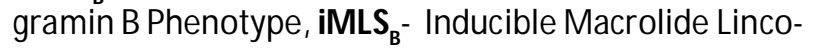
samide Streptogramin B Phenotype.

\section{CONCLUSION}

As a routine diagnostic practice, we go on reporting S.aureus isolates as susceptible to Clindamycin on the basis of Standard disc diffusion method without incorporating any test to check for the inducible Clindamycin resistance and such inappropriate reporting may miss a significant proportion of resistant cases resulting in irrational Clindamycin therapy of such infections leading to treatment failure.We can avoid this simply by incorporating a test like D-test which is very simple, easy and cost effective for detecting inducible Clindamycin resistance amongst Staphylococcal isolates.

We therefore strongly recommend that each and every M icrobiology Laboratory should include Dtest in routine antibiotic susceptibility testing for S.aureus which will enable the judicious and rational use of Clindamycin.

\section{ABBREVIATIONS}

D-Test: Disc Approximation Test

MRSA- Methicillin Resistant Staphylococcus aureus

MSSA-M ethicillin Sensitive Staphylococcus aureus $\mathbf{C M L S}_{\mathbf{B}}$ - Constitutive Macrolide Lincosamide Streptogramin B Phenotype

$\mathbf{i M L S}_{\mathbf{B}}$ - Inducible Macrolide Lincosamide Streptogramin B Phenotype

ATCC- American Type Culture Collection

CLSI- Clinical and Laboratory Standards Institute

\section{ACKNOWLEDGEM ENT}

We are thankful to Mr.Avanish Upadhyay, Laboratory technician,Dept. of M icrobiology for the technical assistance received.

\section{REFERENCES}

[1]. Prabhu K, Rao S, Rao V. Inducible Clindamycin Resistance in Staphylococcus aureus isolated from Clinical Samples. J Lab Physicians 2011;3(1):25-27. doi: 10.4103/0974-2727.78558

[2]. Deotale V, M endiratta DK, Raut U, Narang P. Inducible clindamycin resistance in Staphylococcus aureus isolated from clinical samples. Indian J Med Microbiol. 2010;28:124-6.

[3]. Sasirekha B, Usha MS, Amruta JA, Ankit S, Brinda $\mathrm{N}$, Divya R. Incidence of constitutive and inducible clindamycin resistance among hospitalassociated Staphylococcus aureus. Biotech. 2014;4(1):85-89. doi: 10.1007/s13205-013-0133-5.

[4]. Ajantha GS, Kulkarni RD, Shetty J, Shubhada C, Jain P. Phenotypic detection of inducible clindamycin resistance among Staphylococcus aureus isolates by using the lower limit of recommended inter-disk distance. Indian J Pathol Microbiol. 2008;51:3768.

[5]. Gupta V, Datta P, Rani H, Chander J. Inducible clindamycin resistance in Staphylococcus aureus: $A$ study from North India. J Post grad Med. 2009; 55:176-9.

[6]. Baiu SH, Al-Abdli NE.Inducible Clindamycin Resistancein Methicillin resistant Staphylococcus aureus. Am J Infect.Dis. Microbiol 2016; 4(1): 25-27.

[8]. CLSI. Performance Standards for Antimicrobial Susceptibility Testing: Twenty Fourth Informational Supplement.CLSI document M 100-S24. Wayne, PA:Clinical and Laboratory Standards Institute;2014.

[9]. Mittal V, Kishore S, Siddique ME. Prevalence of clindamycin resistance among clinical isolates of Staphylococcus aureus by phenotypic method: a preliminary report.J Infect Dis Immun. 2013;5(1):10-12.

[10]. Ghasemian A; Peerayeh SN Bakhshi B Mirzaee $M$. Inducible Clindamycin Resistance in Clinical Isolates of Staphylococcus aureus. Archives of Clinical Infectious Diseases 2014;9(2):e16218, DOI: 10.5812 /archcid.16218.

[11]. Singh T, Deshmukh AB, Chitnis V, Bajpai T. Inducible clindamycin resistance among the clinical isolates of Staphylococcus aureus in a tertiary care hospital. Int J Health Allied Sci 2016;5:111-4.

[12]. Yilmaz G, Aydin K, Iskender S, Caylan R, Koksal I. Detection and prevalence of inducible clindamycin resistance in staphylococci. J Med Microbiol. 2007;56:342-5.

[13]. Gadepalli R, Dhawan B, M ohanty S, Kapil A, Das BK, Chaudhry R. Inducible clindamycin resistance in clinical isolates of Staphylococcus aureus. Indian J Med Res. 2006;123:571-573.

[14]. Rahbar M, Hajia M. Inducible clindamycin resistance in Staphylococcus aureus: a cross-sectional report. Pak J Biol Sci. 2007;10:189-192. doi: 10.3923/pjbs.2007.189.192. 